This information is current as of April 25, 2023. 


\section{ORIGINAL RESEARCH \\ Usefulness of MR Imaging for the Assessment of Nonophthalmic Paraclinoid Aneurysms}

\author{
L. Thines \\ J.-Y. Gauvrit \\ X. Leclerc \\ D. Le Gars \\ C. Delmaire \\ J.-P. Pruvo \\ J.-P. Lejeune
}

BACKGROUND AND PURPOSE: The neuroradiologic location of asymptomatic paraclinoid aneurysms is decisive for patient management. In a preliminary study, we designed a paraclinoid MR protocol (PMP) including high-resolution T2-weighted images in 2 orthogonal planes to define the inferior limit of the distal dural ring plane that represents the borderline between the intradural and extradural internal carotid artery. In this clinical study, we compared this protocol with digital subtraction angiography (DSA) for the location of paraclinoid aneurysms.

MATERIALS AND METHODS: During a 3-year period, we performed PMP and conventional angiograms in 14 consecutive patients with 17 asymptomatic paraclinoid aneurysms. Ophthalmic (superior) aneurysms were excluded. Two independent observers reviewed MR imaging data, and a third experienced neuroradiologist analyzed the conventional angiograms. MR imaging and conventional angiograms were independently analyzed, and interpretations obtained with each technique were compared.

RESULTS: PMP allowed correct visualization of the aneurysms in all patients. No significant differences $(P>.05)$ were found between the DSA and PMP for the measurement of the aneurysmal neck or sac. Interobserver agreement was good. MR imaging was discordant with conventional angiography regarding the position around the cavernous sinus of the aneurysmal neck and sac in 5 cases. PMP images were helpful for treatment decisions in 4 cases.

CONCLUSION: PMP is an interesting tool that might be used in association with conventional angiography for the assessment of paraclinoid aneurysms.
$\mathbf{P}$ araclinoid aneurysms (PA) represent only $5 \%$ to $11 \%$ of asymptomatic intracranial aneurysms. ${ }^{1-5}$ The main goal of the treatment is to prevent subarachnoid hemorrhage (SAH) from aneurysmal rupture, which is associated to high rates of morbidity and mortality. ${ }^{6}$ Asymptomatic extradural (intracavernous) aneurysms are at lower risk of subarachnoid hemorrhage than transitional or intradural aneurysms (1.4\% of SAH and exceptional cataclysmic epistaxis). ${ }^{7}$ However, they can result in mass effect (cranial neuropathies), carotid cavernous fistulas, or distal embolization when partly fusiform. Conversely, the rupture of an intradural (supracavernous) aneurysm is life threatening. The precise location of PA on both sides of the roof of the cavernous sinus is of great importance for patient management. The distal dural ring is the anatomic limit between the intracavernous and supracavernous internal carotid artery (ICA). Unfortunately, the distal dural ring is not easily identifiable because of its small size. We developed a paraclinoid MR protocol (PMP), which allows us to estimate the position of the distal dural ring plane. This protocol was validated previously with use of correlations between a cadaveric model and MR imaging. ${ }^{8,9}$ Our study compares the PMP with digital subtraction angiography (DSA) in clinical conditions.

\section{Materials and Methods}

\section{Patients and Selection of Aneurysms}

We evaluated PMP in 14 consecutive female patients (median age, $45 \pm$ 14 years) harboring 17 asymptomatic PA between June 2002 and June

Received December 7, 2006; accepted after revision May 13, 2007.

From the Departments of Neurosurgery (L.T., J.-P.L.) and Neuroradiology (J.-Y.G., X.L., C.D., J.-P.P.), Centre Hospitalier Régional et Universitaire, Lille, France; Departments of Neurosurgery and Anatomy (D.L.G.), Centre Hospitalier Régional et Universitaire, Amiens, France.

Please address correspondence to Dr. Laurent Thines, Clinique Neurochirurgicale, Hôpital Roger-Salengro, CHRU, 59037 Lille Cedex, France; e-mail: laurent.thines@wanadoo.fr DOI 10.3174/ajnr.A0734
2005. PA were defined as aneurysms arising from the C4, C5, or C6 segments according to the Bouthillier classification of the ICA segments. ${ }^{10}$ We studied carotid cave projecting aneurysms (arising from the medial wall of the ICA, most often at the level of the origin of the superior hypophyseal artery), high-located, cavernous, subclinoid, and low-located posterior carotid aneurysms. Giant aneurysms and purely anterior (superior or "carotid-ophthalmic") aneurysms were excluded.

\section{Angiographic Technique and Classification}

We performed conventional angiograms on an Integris Allura V5000 (Philips Medical Systems, Best, the Netherlands) using the PROPELLER rotation technique. ${ }^{11}$ We then performed postprocessing of dynamic angiographic data, including volume rendering, using the Integris workstation (Philips Medical Systems).

The orientation of the aneurysmal neck and sac was determined according to the angiographic classification described by De Jesus et $\mathrm{al}^{2}$ as follows: we distinguished medial or lateral projections on the anterior view and anterior or posterior projections on the lateral view. To locate on DSA the aneurysmal neck and sac in comparison with the roof of the cavernous sinus and to ensure the reproducibility of the method, we chose the limit represented by the horizontal line passing through the origin of the ophthalmic artery on the lateral and anterior views. We classified the aneurysmal neck and sac as supracavernous, above the ophthalmic line; transitional, crossing the ophthalmic line; and intracavernous, beneath the ophthalmic line. Cases with an obvious intracavernous origin of the ophthalmic artery were excluded.

\section{Paraclinoid MR Protocol}

Brain MR imaging was performed on a $1.5 \mathrm{~T}$ MR system (Vision; Siemens, Erlangen, Germany). A turbo spin-echo T2-weighted sequence (TR, $5000 \mathrm{~ms}$; TE, $120 \mathrm{~ms}$; NEX, 2; contiguous section thickness, $2.0 \mathrm{~mm}$; FOV, $32 \mathrm{~cm}$; matrix, $512 \times 512$ ) was performed. For each aneurysm, we obtained sections in 2 planes: a coronal plane perpendicular to the diaphragma sellae (so-called diaphragmatic 

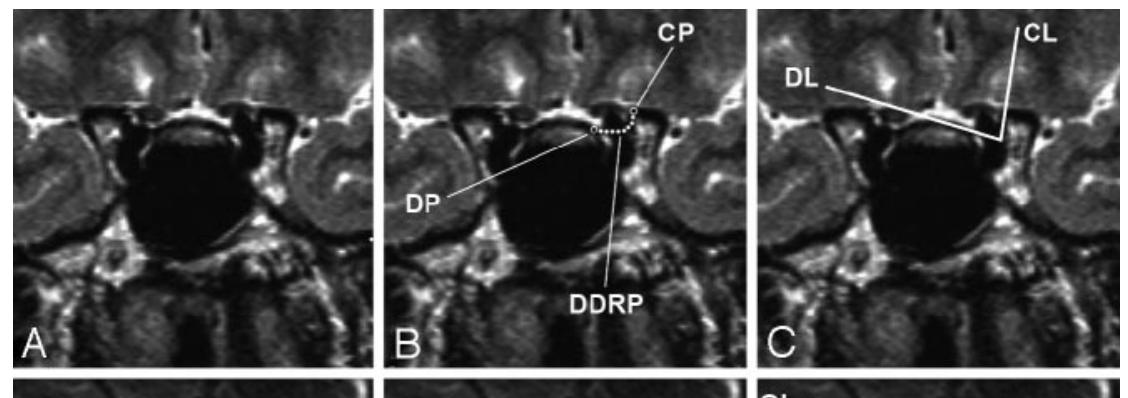

Fig 1. Paraclinoid MR protocol in a control subject showing the 4 reference points. The 4 lines and their angles of intersection correlated with the limits of the distal dural ring plane in each cutting plane. $A, B, C$, Paraclinoid region in the diaphragmatic plane. $D, E, F$, Paraclinoid region in the carotid plane. DP indicates diaphragmatic point; $\mathrm{CP}$, clinoid point; DL, diaphragmatic line; $C L$, clinoid line, $A C N E$, anterior CSF notch extremity; PCNE, posterior CSF notch extremity; $\mathrm{OL}$, optic line; $\mathrm{RL}$, roof line, DDRP, distal dural ring plane (dotted curve).
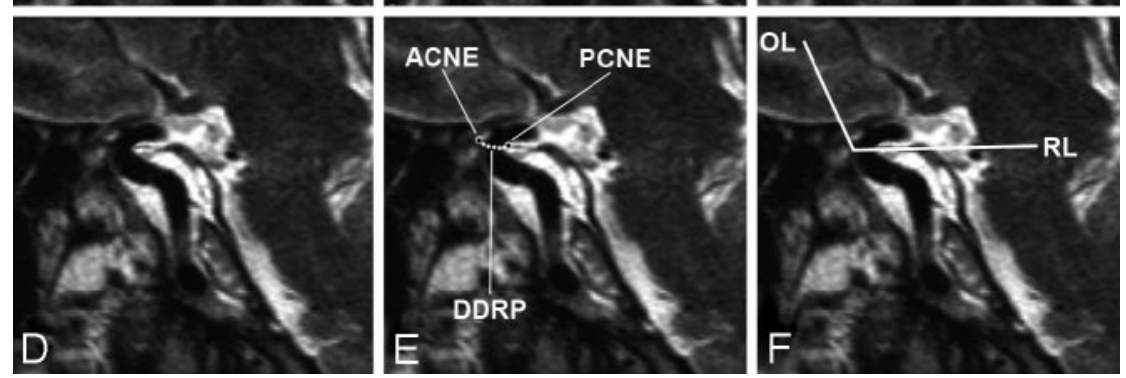

plane) and a sagittal oblique plane, strictly anteroposterior passing through the axis of the carotid siphon (so-called carotid plane).

In preliminary studies, we defined the reference points, correlation lines, and angles that correlated with the limits of the distal dural ring plane in both cutting planes. ${ }^{8,9}$ We used 40 paraclinoid regions (dissected from 20 formalin-fixed human cadaveric heads) to correlate the MR data with a cadaveric model. In our study, the PMP angles allowed us to accurately locate the distal dural ring plane in the diaphragmatic and carotid planes in $70 \%$ and $80 \%$ of the cases, respectively.

In our study, we propose a simple and practical method allowing the use of the PMP protocol in clinical practice (Fig 1). The distal dural ring plane is a curved plane passing through 4 radiologic reference points: the diaphragmatic point (in the diaphragmatic plane) at the borderline between the diaphragma sellae and the roof of the cavernous sinus; the clinoid point (in the diaphragmatic plane) at the medial ridge of the superior surface of the anterior clinoid process; the anterior and posterior CSF notches extremity (in the carotid plane); and indirect markers formed by CSF cisterns around the ICA, respectively, between the optic nerve or the roof of the cavernous sinus and the anterior or posterior aspects of the ICA. The inferior limit of the distal dural ring plane is estimated in each cutting plane by the intersection angle formed by the lines passing through these 4 points. Rapid and simple drawing of those 4 lines can be achieved as follows: diaphragmatic line (through the diaphragmatic point); clinoid line (through the clinoid point); and roof line (through the posterior CSF notch extremity), respectively, tangential to the diaphragma sellae, the inner dural surface of the anterior clinoid process and the cavernous sinus roof, and optic line (through the anterior CSF notch extremity) perpendicular to the optic nerve at the level of its penetrating point in the optic canal.

We determined the orientation of the aneurysmal neck and sac compared with the ICA by distinguishing medial or lateral projections in the diaphragmatic plane, and anterior or posterior projections in the carotid plane.

The MR positioning of the aneurysmal neck and sac compared with the roof of the cavernous sinus was based on their relationship with each intersection angle in both planes as follows: supracavernous (above both intersection angles), transitional (crossing 1 or both in- tersection angles), and intracavernous (beneath both intersection angles).

\section{Treatment of the Aneurysms}

All cases of asymptomatic aneurysms at our institution are managed by a multidisciplinary neurovascular team. For PA, we took into account principally the location of the aneurysmal sac, the history of a previous $\mathrm{SAH}$, and the ages of the patients. When the sac was intracavernous, we chose conservative management. For a transitional or supracavernous sac, embolization or surgery was usually required because of the risk of hemorrhage. An exception was made for some of the transitional or supracavernous aneurysms that were too small $(<1-2 \mathrm{~mm})$ for endovascular or surgical treatment, or for those aneurysms associated with carotid dysplasia.

\section{Descriptive Analysis}

Epidemiologic data for each patient were documented. Angiograms were reviewed on films from anterior, lateral, and 3D views by an experienced neuroradiologist specialized in endovascular treatment of the cerebral aneurysms (X.L.). MR imaging data were reviewed independently by 2 investigators (L.T., J.-Y.G.). We measured the ratio of the neck and sac of each aneurysm and assessed the projection of the aneurysmal neck and sac around the ICA (anterior, posterior, medial, lateral) and their locations regarding the roof of the cavernous sinus. Therapeutic modalities of the management for each patient were recorded. For patients in whom an operation was performed, we took operative field shots to make radiologic-surgical correlations.

\section{Statistical Analysis}

The angiographic and MR imaging results in terms of measurement of the sac and neck, and the sac-to-neck ratio were compared with the $t$ test. The intrinsic validity of the PMP was evaluated with aneurysmal measurement (sac, neck, and sac-to-neck ratio) with the $t$ test and interobserver agreement for the aneurysmal projection around the ICA and location around the roof of the cavernous sinus with the $\kappa$ coefficient. The agreement was considered as low, moderate, good, or excellent according to the respective $\kappa$ coefficient values: $\leq 0.40,0.41-$ $0.60,0.61-0.80, \geq 0.81$. A $P$ value $<.05$ was regarded as significant. 


\section{Results}

\section{General Data}

Fourteen patients (17 PA) were enrolled in the study. After angiography, most patients were found to have at least 1 additional vascular disease: 10 patients with at least 1 associated aneurysm (1 dysplasia) and 1 patient each with contralateral Moyamoya disease and contralateral arteriovenous malformation. Seventeen associated aneurysms were found: carotidophthalmic (5), posterior carotid (5), PA (3), basilar (2), middle cerebral artery (1), and proximal ICA (1). Ten PA were incidentally discovered, and 7 were diagnosed after SAH as a result of an associated vascular disease.

\section{Angiographic Results}

We performed DSA with 3D-reconstructions on most of the PA (14/17). The mean sizes of the necks and sacs were $3.1 \pm 1$ $\mathrm{mm}$ and $5.9 \pm 4 \mathrm{~mm}$, respectively. The mean sac-to-neck ratio was $1.9 \pm 0.9$. Seven PA were located in the intracavernous position, 8 were transitional, and 2 were supracavernous.

\section{MR Imaging Results}

The mean sizes of the necks and sacs were $3.5 \pm 1.3$ to $1.4 \mathrm{~mm}$ and 6.2 to $6.3 \pm 3.8 \mathrm{~mm}$, respectively. The mean sac-to-neck ratio was $1.8 \pm 0.7$. Six PA were located in an intracavernous position, 7 were transitional, and 4 were supracavernous. No significant differences $(P>.05)$ were found between the investigators for the measurement of the aneurysmal neck or sac. The interobserver agreement for the projections of the aneurysmal neck or sac $(\kappa=0.87: 1)$ and for the location of PA around roof of the cavernous sinus $(\kappa=1)$ were "excellent."

\section{Treatment of the Aneurysms}

Ten PA including 6 intracavernous and 4 transitional or supracavernous - but very small $(<2 \mathrm{~mm})$ or associated with severe intracranial arterial dysplasia-were managed conservatively. Six patients with PA underwent endovascular treatment because of the supracavernous (2 cases) and transitional (4 cases) locations of the sac in young patients or in patients with previous SAH. One patient underwent direct surgical treatment of PA.

\section{Surgical Results}

A young patient was operated on after failure of coil embolization for a supracavernous aneurysm, which was revealed by the rupture of an associated aneurysm (case 14). Another patient was operated on for a carotid-ophthalmic aneurysm (superior location) associated with an intracavernous PA (case 16). In both cases, the aneurysmal locations provided by the PMP were similar to the surgical findings. The first aneurysm was supracavernous and was clipped (Fig 2). The second was not seen after full dissection of the paraclinoid region down to the distal dural ring and was supposed to be intracavernous.

\section{Comparison between the PMP and DSA}

No significant difference $(P>.05)$ was found between DSA and the PMP for the measurement of the aneurysmal necks or sacs (Table). In 9 cases (2, 5, 7-9, 11-13, and 16), DSA and the PMP gave the same aneurysmal location (Figs 3, 4, and 5). Interpretations on DSA in cases 1,3, and 6 were different from
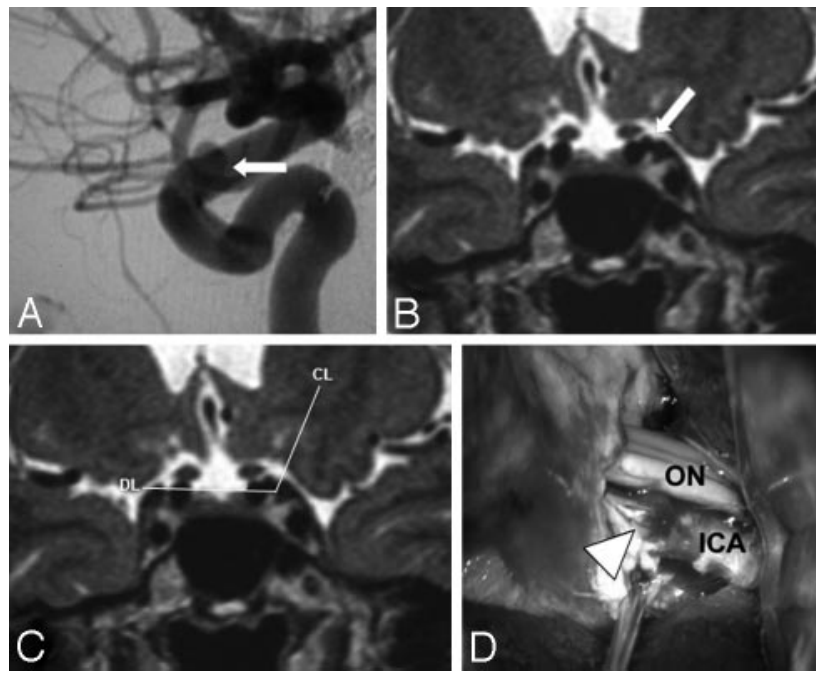

Fig 2. Radiologic-surgical correlation for a left superolateral paraclinoid aneurysm (case 14). A, DSA of the left ICA (lateral view) showing an aneurysm (arrows) interpreted as transitional with an intracavernous neck and a transitional sac. B, C, PMP in the diaphragmatic plane with an intersection angle showing a supracavernous location of the neck and the sac (DL indicates diaphragmatic line; $C L$, clinoid line). D, Operative findings confirming the supracavernous location of both neck and sac and showing, after anterior clinoidectomy and opening of the falciform ligament, the aneurysmal (arrowhead) relationship between the ICA and the optic nerve (ON).

the PMP for the locations of the neck or sac. Interpretations on DSA in cases 4, 10, 14, 15, and 17 were different from the PMP for the locations of the neck and sac (Fig 2).

\section{Discussion}

\section{Review of the Literature}

The origin of the ophthalmic artery is commonly used as the angiographic limit between the intracavernous and supracavernous ICA. ${ }^{4}$ Because of its interindividual variability, ${ }^{4,12-16}$ this reliable marker may sometimes fail to locate this junction precisely, anatomically represented by the distal dural ring.

\begin{tabular}{|c|c|c|c|c|c|c|}
\hline \multicolumn{7}{|c|}{$\begin{array}{l}\text { Comparison between DSA and the PMP for the location of } \\
\text { paraclinoid aneurysms around the roof of the cavernous sinus }\end{array}$} \\
\hline \multirow[b]{2}{*}{ Aneurysms } & \multicolumn{2}{|c|}{$\begin{array}{l}\text { Aneurysmal } \\
\text { Projection }\end{array}$} & \multicolumn{2}{|c|}{$\begin{array}{l}\text { Angiographic } \\
\text { Location }\end{array}$} & \multicolumn{2}{|c|}{$\begin{array}{c}\text { PMP } \\
\text { Location }\end{array}$} \\
\hline & Neck & Sac & Neck & Sac & Neck & Sac \\
\hline $1^{*}$ & $\mathrm{MP}^{*}$ & $\mathrm{MP}^{*}$ & $S C^{*}$ & $\mathrm{~T}^{*}$ & $S C^{*}$ & $\mathrm{SC}^{*}$ \\
\hline 2 & $\mathrm{M}$ & $\mathrm{M}$ & $\mathrm{IC}$ & IC & IC & $\mathrm{IC}$ \\
\hline $3^{*}$ & $\mathrm{P}^{*}$ & $\mathrm{P}^{*}$ & $S C^{*}$ & $\mathrm{~T}^{*}$ & $T^{*}$ & $T^{*}$ \\
\hline $4^{*}$ & $M^{*}$ & $M^{*}$ & $\mathrm{IC}^{*}$ & $I C^{*}$ & $\mathrm{SC}^{*}$ & $\mathrm{SC}^{*}$ \\
\hline 5 & $\mathrm{M}$ & $\mathrm{M}$ & $\mathrm{T}$ & $\mathrm{T}$ & $\mathrm{T}$ & $\mathrm{T}$ \\
\hline $6^{*}$ & $P^{*}$ & $\mathrm{P}^{*}$ & $S C^{*}$ & $S C^{*}$ & $S C^{*}$ & $\mathrm{~T}^{*}$ \\
\hline 7 & MP & MP & IC & IC & IC & IC \\
\hline 8 & MP & $\mathrm{MP}$ & IC & IC & IC & IC \\
\hline 9 & $\mathrm{MP}$ & $\mathrm{MP}$ & IC & IC & IC & IC \\
\hline $10^{*}$ & $\mathrm{MP}^{*}$ & $\mathrm{MP}^{*}$ & $\mathrm{~T}^{*}$ & $I C^{*}$ & $S C^{*}$ & $T^{*}$ \\
\hline 11 & $\mathrm{M}$ & MP & SC & $\mathrm{T}$ & SC & $\mathrm{T}$ \\
\hline 12 & $A$ & $\mathrm{~A}$ & SC & $\mathrm{SC}$ & SC & SC \\
\hline 13 & $\mathrm{MP}$ & $\mathrm{MP}$ & IC & $\mathrm{T}$ & IC & $\mathrm{T}$ \\
\hline $14^{*}$ & $\mathrm{LA}^{*}$ & $L^{*}$ & $I C^{*}$ & $\mathrm{~T}^{*}$ & $S C^{*}$ & $S C^{*}$ \\
\hline $15^{*}$ & $\mathrm{MP}^{*}$ & $M^{*}$ & $I C^{*}$ & $I C^{*}$ & $\mathrm{~T}^{*}$ & $T^{*}$ \\
\hline 16 & $\mathrm{MP}$ & $P$ & IC & IC & IC & IC \\
\hline $17^{*}$ & $M^{*}$ & $\mathrm{MP}^{*}$ & $T^{*}$ & $\mathrm{~T}^{*}$ & $I C^{*}$ & $\mathrm{IC}^{*}$ \\
\hline
\end{tabular}

Note:- $M$ indicates medial; $L$, lateral; $A$, anterior; $P$, posterior; $I C$, intracavernous; $T$ transitional; SC, supracavernous.

* Cases with different interpretations. 

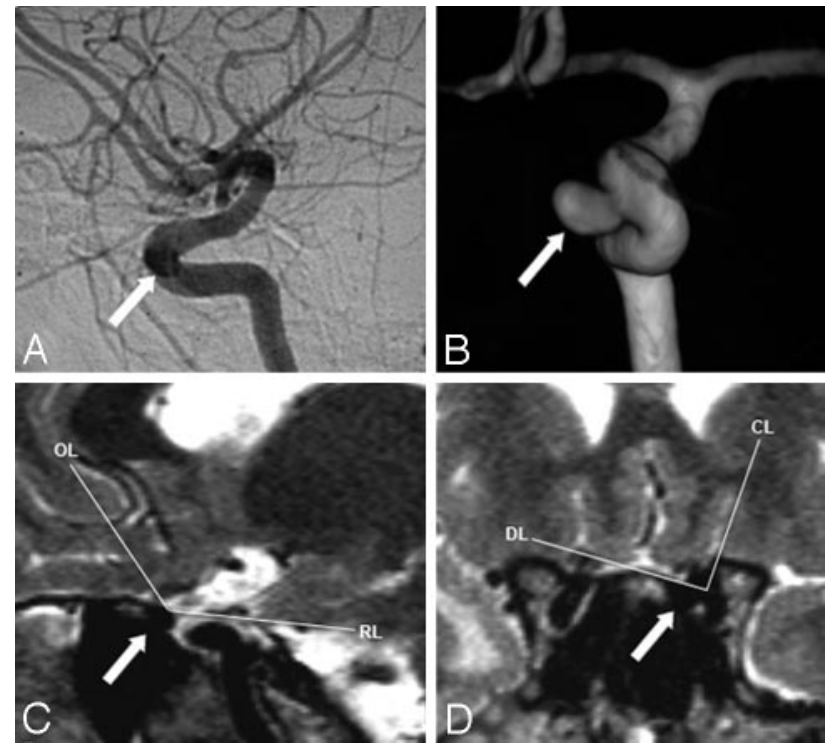

Fig 3. Comparison between angiogram and PMP for the location of case 2 (arrows). Both examinations show an intracavernous aneurysm (neck and sac intracavernous). $A$, DSA of the left ICA in the lateral view. B, 3D-DSA of the left ICA in the anterior view. C, PMP in the carotid plane (OL indicates optic line; $\mathrm{RL}$, roof line). $D, \mathrm{PMP}$ in the diaphragmatic plane (DL indicates diaphragmatic line; CL, clinoid line).
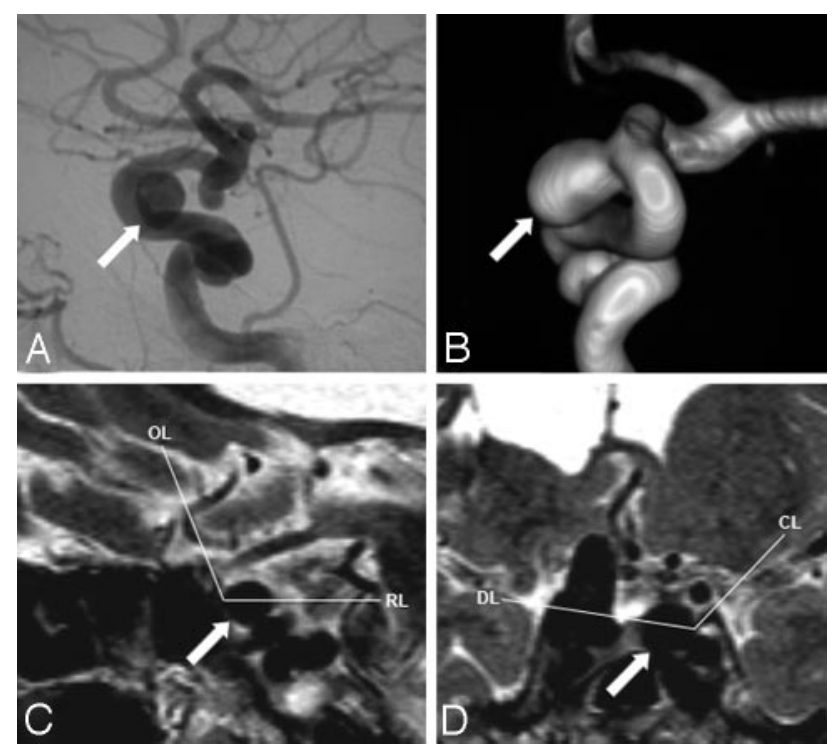

Fig 4. Comparison between angiogram and the PMP for the location of case 13 (arrows). Both examinations show a transitional aneurysm (neck intracavernous and sac transitional). The PMP depicts a widest supracavernous portion than it is suspected on initial DSA. A, DSA of the left ICA in the lateral view. B, 3D-DSA of the left ICA in the anterior view. $C$, PMP in the carotid plane (OL indicates optic line; $\mathrm{RL}$, roof line). D, PMP in the diaphragmatic plane (DL indicates diaphragmatic line; $\mathrm{CL}$, clinoid line).

The ophthalmic artery may arise from the ICA in the extradural space, most often far below the distal dural ring (artery of the foramen rotundum, inferolateral trunk) and is easily recognized as anomalous and unreliable but rarely near the distal dural ring (clinoid space, interdural location). Most ophthalmic arteries originate in the intradural space, but the distance between the origin and the distal dural ring may vary in $24 \%$ to $50 \%$ of cases. This variability led some to design techniques of radiographic correlation using some of the bony structures of the skull base on lateral and anterior nonsubtracted angiograms: the base of the anterior clinoid process, ${ }^{17}$
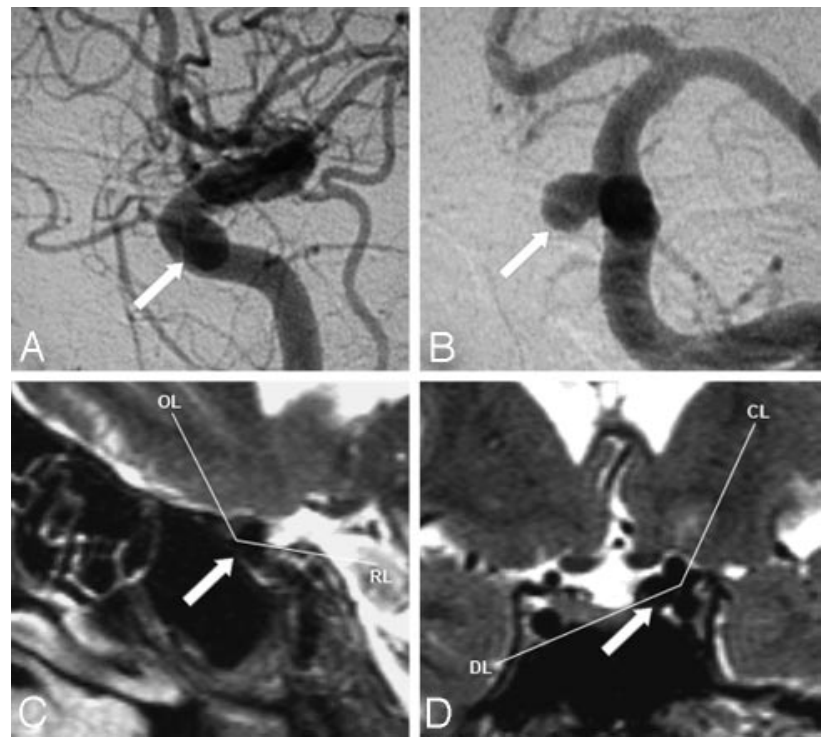

Fig 5. Comparison between angiogram and PMP for the location of case 11 (arrows). Both examinations show a transitional aneurysm (neck supracavernous and sac transitional). The PMP depicts a wider supracavernous portion than is suspected on initial DSA. A, DSA of the left ICA in the lateral view. $B, D S A$ of the left ICA in the anterior view. $C$, PMP in the carotid plane (OL indicates optic line; $\mathrm{RL}$, roof line). $D, P M P$ in the diaphragmatic plane (DL indicates diaphragmatic line; CL, clinoid line).

the superior surface of the anterior clinoid process and the tuberculum sellae ${ }^{18}$ or the planum sphenoidale, and lines of the tuberculum sellae. ${ }^{16}$ Recently, $3 \mathrm{D}$ reconstructions from CT scans, ${ }^{19} \mathrm{CT}$ angiograms,${ }^{20}$ or contrast medium-enhanced tomographic cisternograms ${ }^{21}$ have been used. These radiologic procedures were respectively based on the visualization of the strut of the optic canal, identification of a surface concavity at the level of the distal dural ring, and CSF cisterns contrast around the ICA. Although effective, these protocols did not take into account the complexity of the extravascular anatomy of the paraclinoid region, particularly of the radiotransparent dural folds of the walls of the cavernous sinus. An indirect approach and interindividual radiologic variability were the major limitations of the above techniques.

\section{Value and Limitations of the PMP}

Reproducibility and accuracy of the PMP was proved in preliminary anatomoradiologic studies. ${ }^{8,9}$ In this clinical study, the MR technique allowed the identification and the precise positioning of the PA detected with DSA for all patients. The results confirmed the interobserver reproducibility for the aneurysmal measurement and location, and the reliability of the aneurysmal dimensions obtained with the PMP. In 2 cases, the results of the PMP were confirmed by surgical exploration of the paraclinoid region. Compared with DSA, the PMP provides a global view of the paraclinoid anatomy and direct visualization of the radiotransparent dural folds comprising the roof of the cavernous sinus. Therefore, the PMP could increase the quality of the overall radiologic analysis by providing direct visualization of the aneurysm and a reliable evaluation of the size of the intracavernous and supracavernous portions of transitional aneurysms.

Discordance between interpretations of DSA and PMP were found in 8 cases. Differences were more frequent for medial or medial-posterior projecting PA (5/8) and for the location of the 
aneurysmal sacs (7/8). Improvement of the location of the aneurysmal sac around the roof of the cavernous sinus is of importance, considering that it is the most usual site of rupture. In our study, we had surgical correlations in 2 cases only, so the discordances between DSA and the PMP should be carefully analyzed. Furthermore, the arbitrary choice of the horizontal line passing through the origin of the ophthalmic artery as the only angiographic limit between the intracavernous and extracavernous spaces could have led us to misinterpret some of the DSA. Some aneurysms could have been quoted in the wrong location. In those cases, the PMP technique has perhaps helped us only to clarify misinterpretations on DSA.

This PMP technique requires a short training period to become familiar with the MR imaging anatomy of the paraclinoid region. Therefore, a simplified protocol that could be applied in clinical practice by all neuroradiologists is proposed.

\section{Potential Implications for the Management of PA}

Advances in neuroradiologic techniques are responsible for the increased diagnosis of asymptomatic aneurysms. Furthermore, because of therapeutic implications, clinicians' and patients' demands for radiologic accuracy have grown. The location of PA compared with the roof of the cavernous sinus illustrates this situation perfectly. In the treatment decision making, the neurovascular team takes into account the benefit-to-risk ratio among 3 therapeutic options: conservative, endovascular, or surgical. The rates of morbidity and mortality related to endovascular and surgical treatment of asymptomatic aneurysms are, respectively, $1 \%$ and $4 \%{ }^{6,22}$ and $2.6 \%$ and $10.9 \%,{ }^{22,23}$ the surgical morbidity being slightly higher for PA. ${ }^{24}$ The spontaneous risk of rupture of these aneurysms (sizes are approximately $6 \mathrm{~mm}$ and are located on the anterior circulation) varies between $0.05 \%$ and $0.5 \%$ per year, whether there is a previous history of SAH from an associated aneurysm (7/17 cases in this study). ${ }^{25}$ In women with a mean age of 45 years and a life expectancy of approximately 84 years, the cumulative risk of rupture (conservative treatment) can reach $1.8 \%$ to $18 \%$ and is not so different from the risk of endovascular or surgical treatment, especially if there are individual risks of aneurysmal growth such as arterial hypertension, smoking, or a familial history of SAH or connective tissue disease. ${ }^{6,26-28}$ The decision of curative treatment for PA relies on the certainty that they are supracavernous or transitional aneurysms (at risk of $\mathrm{SAH})$.

In our own clinical experience, the PMP allowed us to differentiate among intracavernous, supracavernous, and transitional aneurysms more precisely and to adapt the therapeutic management to the aneurysmal location. Intracavernous aneurysmal sacs were conservatively managed, whereas supracavernous or transitional aneurysmal sacs were treated with endovascular or surgical intervention. Only very small or dysplastic supracavernous or transitional sacs were not treated. Overall, the association of DSA and PMP benefited the treatment decisions in 4 patients (cases 10, 14, 15, and 17).

\section{Conclusions}

The paraclinoid MR protocol is a noninvasive and nonradiating alternative technique, complementary to DSA. It is efficient for the location around the roof of the cavernous sinus of small and asymptomatic PA. This protocol might help to provide patients with more appropriate information about their disease and therapeutic solutions adjusted in relationship to the potential risk of their aneurysm(s).

\section{References}

1. Day AL. Aneurysms of the ophthalmic segment. A clinical and anatomical analysis. J Neurosurg 1990;72:677-91

2. De Jesũs O, Sekhar LN, Riedel CJ. Clinoid and paraclinoid aneurysms: surgical anatomy, operative techniques, and outcome. Surg Neurol 1999;51:477-87; discussion 487-88

3. Gurian JH, Viñuela F, Guglielmi G, et al. Endovascular embolization of superior hypophyseal artery aneurysms. Neurosurgery 1996;39:1150-54; discussion $1154-56$

4. Punt J. Some observations on aneurysms of the proximal internal carotid artery. J Neurosurg 1979;51:151-54

5. Knosp E, Muller G, Perneczky A. The paraclinoid carotid artery: anatomical aspects of a microneurosurgical approach. Neurosurgery 1988;22:896-901

6. Wardlaw JM, White PM. The detection and management of unruptured intracranial aneurysms. Brain 2000;123:205-21

7. Kupersmith MJ, Hurst R, Berenstein A, et al. The benign course of cavernous carotid artery aneurysms. J Neurosurg 1992;77:690-93

8. Thines L, Delmaire C, Le Gars D, et al. MRI location of the distal dural ring plane: anatomoradiological study and application to paraclinoid carotid artery aneurysms. Eur Radiol 2006;16:479-88

9. Thines L, Delmaire C, Le Gars D, et al. [MRI localization of paraclinoid carotid aneurysms]. J Neuroradiol 2006;33:115-20

10. Bouthillier A, van Loveren HR, Keller JT. Segments of the internal carotid artery: a new classification. Neurosurgery 1996;38:425-32; discussion 432-33

11. Gauvrit JY, Leclerc X, Vermandel M, et al. 3D rotational angiography: use of propeller rotation for the evaluation of intracranial aneurysms. AJNR Am J Neuroradiol 2005;26:163-65

12. Huynh-Le P, Natori Y, Sasaki T. Surgical anatomy of the ophthalmic artery: its origin and proximal course. Neurosurgery 2005;57(4 suppl):236-41; discussion 236-41

13. Kim JM, Romano A, Sanan A, et al. Microsurgical anatomic features and nomenclature of the paraclinoid region. Neurosurgery 2000;46:670-80; discussion $680-82$

14. Kyoshima K, Oikawa S, Kobayashi S. Interdural origin of the ophthalmic artery at the dural ring of the internal carotid artery. Report of two cases. J Neurosurg 2000;92:488-89

15. Renn WH, Rhoton AL. Microsurgical anatomy of the sellar region. J Neurosurg 1975;43:288-98

16. Beretta $F$. The paraclinoid aneurysms and the distal dural ring: a new classification. J Neurosurg Sci 2004;48:161-75

17. Taptas JN. Intradural and extradural ICA. J Neurosurg 1979;51:877-78

18. Oikawa S, Kyoshima K, Kobayashi S. Surgical anatomy of the juxta-dural ring area. J Neurosurg 1998;89:250-54

19. Gonzalez LF, Walker MT, Zabramski JM, et al. Distinction between paraclinoid and cavernous sinus aneurysms with computed tomographic angiography. Neurosurgery 2003;52:1131-37; discussion 1138-39

20. Murayama Y, Sakurama K, Satoh K, et al. Identification of the carotid artery dural ring by using three-dimensional computerized tomography angiography. Technical note. J Neurosurg 2001;95:533-36

21. Ito K, Hongo K, Kakizawa Y, et al. Three-dimensional contrast medium-enhanced computed tomographic cisternography for preoperative evaluation of surgical anatomy of intradural paraclinoid aneurysms of the internal carotid artery: technical note. Neurosurgery 2002;51:1089-92; discussion 1092-93

22. Molyneux A, Kerr R, Stratton I, et al. International Subarachnoid Aneurysm Trial (ISAT) of neurosurgical clipping versus endovascular coiling in 2143 patients with ruptured intracranial aneurysms: a randomised trial. Lancet 2002;360:1267-74

23. Raaymakers TW, Rinkel GJ, Limburg M, et al. Mortality and morbidity of surgery for unruptured intracranial aneurysms: a meta-analysis. Stroke 1998;29:1531-38

24. Kumon Y, Sakaki S, Kohno K, et al. Asymptomatic, unruptured carotid-ophthalmic artery aneurysms: angiographical differentiation of each type, operative results, and indications. Surg Neurol 1997;48:465-72

25. Unruptured intracranial aneurysms-risk of rupture and risks of surgical intervention. International Study of Unruptured Intracranial Aneurysms Investigators. N Engl J Med 1998;339:1725-33

26. Broderick JP, Viscoli CM, Brott T, et al. Major risk factors for aneurysmal subarachnoid hemorrhage in the young are modifiable. Stroke 2003;34:1375-81

27. Qureshi AI, Sung GY, Suri MF, et al. Factors associated with aneurysm size in patients with subarachnoid hemorrhage: effect of smoking and aneurysm location. Neurosurgery 2000;46:44-50

28. Raaymakers TW, Rinkel GJ, Ramos LM. Initial and follow-up screening for aneurysms in families with familial subarachnoid hemorrhage. Neurology 1998;51:1125-30 